\title{
TNF- $\alpha$ plus IL-1 $\beta$ Induces opposite regulation of hemichannels and gap junctions in mesangial cells through a RhoA/ROCK-dependent pathway
}

Claudia M. Lucero ${ }^{1}$, Marcelo León ${ }^{1}$, Paola Fernández ${ }^{2}$, Juan A. Orellana ${ }^{4}$, Victoria Velarde ${ }^{3}$, Juan C. Sáez ${ }^{2,3,{ }^{*}}$ and Gonzalo I. Gómez ${ }^{1, *}$

1. Instituto de Ciencias Biomédicas, Facultad de Ciencias de la Salud, Universidad Autónoma de Chile, El Llano Subercaseaux \#2801, Santiago 8910060, Chile; claulucerom@gmail.com (C.M.L.); marceloleonkine@hotmail.com (M.L.)

2. Instituto Milenio, Centro Interdisciplinario de Neurociencias de Valparaíso, Universidad de Valparaíso, 2381850, Valparaíso, Chile; paolafernandezbq@gmail.com (P.F.)

3. Departamento de Fisiología, Facultad de Ciencias Biológicas, Pontificia Universidad Católica de Chile, Alameda \#340, 8331150, Santiago, Chile; vvelarde@bio.puc.cl (V.V.)

4. Departamento de Neurología, Escuela de Medicina y Centro Interdisciplinario de Neurociencias, Facultad de Medicina, Pontificia Universidad Católica de Chile, Santiago 8330024, Chile; jaorella@uc.cl (J.A.O.)

* Correspondence: gonzalo.gomez@uautonoma.cl (G.I.G.); jcsaez@uc.cl (J.C.S.). Tel.: +56-2-2354-6185 (G.I.G.); +56-2-2354-2860 (J.C.S.)

\begin{abstract}
Connexin 43 (Cx43) is expressed in kidneys and constitutes a feedforward mechanism leading to inflammation in other tissues where they form hemichannels and gap junction channels. However, the possible functional relationship between these membrane channels and their role in damaged renal cells remains unknown. Here, analyses of ethidium uptake and thiobarbituric acid reactive species revealed that TNF- $\alpha$ plus IL- $1 \beta$ increase Cx43 hemichannel activity and oxidative stress in MES-13 cells, a cell line derived from mesangial cells. The latter also was accompanied by a reduction in gap junctional communication, whereas western blotting analysis showed a progressive increase of phosphorylated MYPT (a substrate of RhoA/ROCK) and Cx43 upon TNF- $\alpha / / L-1 \beta$ treatment. Additionally, inhibition of RhoA/ROCK strongly diminished the TNF- $\alpha / \mathrm{LL}-1 \beta$-induced activation of $\mathrm{Cx} 43$ hemichannels and reduction in gap junctional coupling. We propose that
\end{abstract}


activation of $\mathrm{Cx} 43$ hemichannels and inhibition of cell coupling during pro-inflammatory conditions could contribute to oxidative stress and damage of mesangial cells via the RhoA/ROCK pathway.

Keywords: connexin hemichannel; gap junction; oxidative stress; Inflammatory receptors; Fasudil; Y-27632.

\section{Introduction}

Chronic kidney disease (CKD) has been recognized as a leading public health problem worldwide [1-3] and consists in persistent alterations in kidney structure and/or function [3]. Regardless of the initiating insult or disease, the most common pathological manifestation of CKD is renal fibrosis [3]. The latter represents the unsuccessful wound-healing of kidney tissue after chronic and sustained injury that is characterized by glomerulosclerosis, tubular atrophy, and interstitial fibrosis. Glomerulosclerosis occurs due to endothelial damage and dysfunction, the proliferation of smooth-muscle cells and mesangial cells (MCs), and the destruction of podocytes that generally line the glomerular basement membrane [1-3].

Among renal cells, the glomerular MCs share most phenotypical similarities with fibroblasts [4]. As one of the significant matrix-producing cells, MCs can secrete mesangial matrix components, such as type IV and type $V$ collagens and fibronectin, which contribute to the excess extracellular matrix. Moreover, MCs also secrete several inflammatory cytokines, adhesion molecules, chemokines, and enzymes, all of which participate in the progression of renal fibrosis [4]. The primary pathological mechanism linking oxidative stress (OS), inflammation, and CKD progression includes an initial kidney injury caused by intraand extracellular oxygen-derived radicals and the subsequent inflammatory response [5]. Indeed, superoxide and hydroxyl radicals readily interact with the molecular components of nephrons [5]. The tumor necrosis factor- $\alpha$ (TNF- $\alpha$ ), initiates the pro-inflammatory response by binding to its receptors, TNFR1 and TNFR2, expressed on tubular (and other) cell surfaces, triggering pathways that activate the nuclear factor $\kappa \mathrm{B}(\mathrm{NF} \kappa \mathrm{B})$ and downstream signaling [6]. Similar effects have been attributed to IL-1 $\beta$ in human and experimental kidney diseases [7]. Recently, in an experimental model of type II diabetes, the IL-1 $\beta$ receptor antagonist, anakinra, presented protective effects, unveiling the participation of this cytokine in renal inflammation [7]. During kidney injury, MCs are the principal target of angiotensin II (Angll) and participate in inflammatory reactions and OS, which lead to CKD involving the increase in intraglomerular pressure $[8,9]$. Consequently, within the glomerulus, the 
activation of MCs, epithelial cells, and podocytes elicit the release of vasoactive and proinflammatory agents that increase cell damage and promote fibrosis. This not only causes the reduction of renal blood flow, but also leads to deficits in permeability during filtration [10]. Several studies have shown that the inflammatory response requires reactive oxygen species (ROS) to link Angll with downstream production of TNF- $\alpha$, IL-1 $\beta$, and IL-6 [2,9].

The renin-angiotensin system (RAS) is a vital endocrine control system in mammals with Angll being the primary active component [11]. Angll binds to several receptors, including the two specific and ubiquitous cytoplasmic angiotensin membrane G-proteincoupled receptors denominated type I (AT1) and type II (AT2), to carry out many biological functions [12]. RAS is one of the crucial regulatory systems controlling blood pressure and fluid balance and comprises two principal axes: the conventional and non-conventional $[13,14]$. The principal promoter of the RAS conventional axis is Angll, which is released by local and systemic RAS activation further to stimulate its receptor Angll type1 receptor (AT1R). The latter results in renal vasoconstriction and inflammation and tubular damage with significant and consequences for the progression of several kidney diseases. This includes nephropathies, renal artery stenosis, and acute kidney injury [14]. On the other hand, the non-conventional or protective axis of RAS induces vasodilation in various tissues such as the heart, kidney, lung, and blood vessels [13,14].

AT1 and AT2 receptors, both coupled to a G-protein, mediate the actions of Ang II. The AT1 receptor activates small G proteins, including Ras, Rac1, RhoA, and the Rho kinase system (ROCK) [15], while the AT2 receptor inhibits RhoA [16]. One major group of small GTPases, the Rho GTPases (average molecular weight 20-40 kDa), regulate the cell junction, cytoskeleton, and cell migration [11]. RhoA is the most recognized member of the Rho GTPase family. ROCK, which exists in two isoforms, ROCK1 and ROCK2, is a downstream effector of RhoA [17]. ROCK is critical when it comes to controlling migration, proliferation, cell apoptosis/survival, gene transcription, and differentiation [17]. The RhoA/ROCK signaling pathway plays an essential role in cell differentiation, migration, growth, and development [18]. The alteration of this signaling pathway can lead to various diseases, including renal interstitial fibrosis caused by unilateral ureteral obstruction [18]. Regarding a therapeutic approach, Fasudil and Y27632, two non-selective ROCK1/2 inhibitors, have been used to evaluate the role of ROCK in several animal disease models [19]. For example, in a rat heart ischemia-reperfusion, Fasudil reduced the infarct size by attenuating endoplasmic reticulum stress and modulating the activity of sarco/endoplasmic 
reticulum $\mathrm{Ca}^{2+}$-ATPase [19]. However, the role of the RhoA/ROCK pathway in the regulation of adhesion and inflammation in the glomerulus remians unsubstantiated [17].

Inflammation encompases a myriad of complex mechanisms and cellular processes, being dysfunctional communication among cells a critical aspect of it. In vertebrates, part of the intercellular communication takes place through gap junctions (GJs) and hemichannels (HCs) [9]. GJs are clusters of intercellular channels, each resulting from the docking of two $\mathrm{HCs}$ or connexons at cell-cell interfaces. An HC is constituted by the oligomerization of six protein subunits called connexins (Cxs) around a central pore. Thus, GJs communicate the cytoplasm of adjacent cells, whereas undocked HCs allow the diffusion of substances between the cytoplasm and extracellular space [9]. Both GJs and HCs are permeable to ions and small molecules, permitting the coordination and regulation of different biological processes [9]. There are 20 and $21 \mathrm{Cx}$ isoforms in humans and rats, respectively [20], with Cx43 being the main isoform in vascular endothelial cells and that plays a protagonic role in regulating vascular diseases such as atherosclerosis, hypotension, and bradycardia [21]. Several Cxs, including Cx26, Cx30.3, Cx31, Cx32, Cx37, Cx40, Cx43, Cx45, and Cx46, contribute to renal pathophysiology, and half of their members are expressed by various cell types located throughout different renal cellular types [21,22].

Most studies showed have shown an increase in $\mathrm{Cx} 43$, where in vitro data from renal epithelial cells exposed to high glucose provided a functional explanation for Cx43 upregulation under stressful conditions [21]. In particular, this evidence indicates that Cx43 GJs mediate the intercellular transfer of deleterious $\mathrm{Ca}^{2+}$ signals for proper cell function [21]. On the other hand, it was found that MES-13 cells, a line derived from MCs, stimulated with Angll, developed oxidative stress, secreted proinflammatory cytokines (IL-1 $\beta$ and TNF- $\alpha$ ), and showed a progressive increase in the activity of hemichannels. In addition, western blotting analysis showed that phosphorylated MYPT (a substrate of RhoA/ROCK pathway) and $\mathrm{Cx} 43$ increased progressively upon Angll treatment, suggesting a possible relationship between the RhoA/ROCK pathway and Cx43 [9]. However, the precise role of Cx43 in the progression of renal disease remains unclear. Here, we found that TNF- $\alpha$ plus IL-1 $\beta$ increases OS and Cx43 HC activity in MES-13 cells and these effects were prevented by Fasudil or $Y-27632$, both inhibitors of the RhoA/ROCK pathway.

\section{Materials and Methods}

\section{Reagents}


TNF- $\alpha$ and IL-1 $\beta$ were obtained from Alomone (Jerusalem, Israel); Fasudil, Y-27632, ethidium $\left(\mathrm{Etd}^{+}\right)$bromide, lanthanum $\left(\mathrm{La}^{3+}\right)$ chloride, and malondialdehyde (MDA) were obtained from Sigma-Aldrich (St. Louis, MO, USA); Gap27, a peptide that inhibits Cx43 HCs, was obtained from NeoMPS, SA (Strasbourg, France). The monoclonal anti-a-tubulin antibody was purchased from Sigma-Aldrich (St. Louis, MO, USA); the polyclonal antiphosphorylated-MYPT1 (Thr696) antibody was obtained from Merck Millipore (Darmstadt, Germany); the monoclonal anti-MYPT1 antibody was purchased from BD Transduction Laboratories (San José, CA, USA); the monoclonal anti-unphosphorylated Cx43 antibody was obtained from Invitrogen (Carlsbad, CA, USA); anti-mouse and anti-rabbit secondary antibodies conjugated to horseradish peroxidase were from Santa Cruz Biotechnology Inc. (Santa Cruz, CA, USA).

\section{Cell Culture}

The cell line MES-13, derived from mesangial cells (CRL-1927 from ATCC, Manassas, VA, USA), was cultured and treated with TNF- $\alpha$ plus IL-1 $\beta$ (10 ng/ml of each one) for different periods $(0,24,48$ and $72 \mathrm{~h})$ in a 2:1 mixture of DMEM and F-12 tissue culture media supplemented with $100 \mathrm{U} / \mathrm{mL}$ penicillin and $100 \mu \mathrm{g} / \mathrm{mL}$ streptomycin. Cells were kept at $37^{\circ} \mathrm{C}$ in a $5 \% \mathrm{CO}_{2} / 95 \%$ air, at nearly $100 \%$ relative humidity. Fasudil $(15 \mu \mathrm{M})$ or $\mathrm{Y}-27632(15 \mu \mathrm{M})$ were added $24 \mathrm{~h}$ before the end of a three days experiment to cell cultures treated with TNF$\alpha$ and IL-1 $\beta$ at time zero.

\section{Dye Uptake}

The activity of $\mathrm{Cx}$ HCs was evaluated by using the dye uptake method, as previously described [23]. In brief, cells were plated onto glass coverslips and bathed with Locke's saline solution (in mM: $154 \mathrm{NaCl}, 5.4 \mathrm{KCl}, 2.3 \mathrm{CaCl}_{2}, 1.5 \mathrm{MgCl}_{2}, 5 \mathrm{HEPES}, 5$ glucose, and $\mathrm{pH} 7.4)$ containing $5 \mu \mathrm{M}$ ethidium bromide $\left(\mathrm{Etd}^{+}\right)$, a molecule that crosses the plasma membrane through large-pore channels, including HCs [23]. Since Etd ${ }^{+}$fluoresces upon its intercalation between nucleotides of the DNA, time-lapse recordings of fluorescent images were measured (at regions of interest in different cells) every $30 \mathrm{~s}$ for 13 min using a Nikon Eclipse Ti inverted microscope (Tokio, Japan) and NIS-Elements software. The basal fluorescence signal was recorded in cells only in Locke's saline solution that contained divalent cations.

\section{Dye Coupling}


MES-13 cells seeded on glass coverslips $\left(n^{\circ} 1\right)$ were bathed with Locke's saline solution, and then observed using an inverted microscope equipped with an xenon arc lamp and a Nikon B filter (excitation wavelength: 450-490 nm, emission wavelength: above $520 \mathrm{~nm}$ ). $\mathrm{Etd}^{+}(25 \mathrm{mM})$ was microinjected through a glass microelectrode into one cell. Dye transfer to neighboring cells was evaluated two minutes after injection. We routinely performed all dye coupling experiments in the presence of $\mathrm{La}^{3+}(150 \mu \mathrm{M})$ to prevent $\mathrm{Etd}^{+}$leakage through HCs that would reduce the intercellular diffusion among coupled cells [24]. The incidence of coupling corresponded to the percentage of cases where the dye spread occurred to at least one neighboring cell. The coupling index was calculated as the average number of cells to which the dye had spread, divided by the number of positive cases. Intracellular coupling was tested in all experiments by injecting a minimum of 10 cells.

\section{Western Blot Assays}

Cell cultures were placed on ice, washed twice with ice-cold PBS ( $\mathrm{pH} 7.4)$, harvested by scraping in $80 \mu \mathrm{L}$ of a solution containing a protease and phosphatase inhibitor cocktail (Thermo Scientific, Pierce, Rockford, IL, USA; cat \# 78430). Lysates were centrifuged (25,200× g, Eppendorf Centrifuge 5415C, Hamburg, Germany), and supernatants were collected for Western blot analysis. Protein concentration was determined using Lowry's method [25]. Samples of homogenized cell cultures (50 $\mathrm{\mu g}$ of proteins) under different conditions were resolved by electrophoresis in 10\% SDS-polyacrylamide gel, and in one lane pre-stained molecular weight markers were resolved. Proteins were transferred to a PVDF membrane (pore size: $0.45 \mu \mathrm{m}$ ), which was blocked at room temperature with Tris $\mathrm{pH}$ $7.4,5 \%$ skim milk $(\mathrm{w} / \mathrm{v})$ and $1 \% \mathrm{BSA}(\mathrm{w} / \mathrm{v})$. Then, the PVDF membrane was incubated overnight at $4^{\circ} \mathrm{C}$ with anti-Cx43 (1:1000), anti-p-MYPT1 $(1: 500)$, or anti-MYPT1 $(1: 1,000)$ antibody, followed by incubation with rabbit or mouse secondary antibody conjugated to peroxidase (1:2,000 both) for $1 \mathrm{~h}$ at room temperature. Then, the PVDF membrane was stripped and reblotted with the anti- $\alpha$-tubulin antibody $(1: 5,000)$ used as loading control, following the same procedure described above. After repeated rinses, immunoreactive proteins were detected by using ECL reagents (Pierce Biotechnology, Rockford, IL, USA) according to the manufacturer's instructions. The bands detected were digitized and subjected to densitometry analysis using the software Image $\mathrm{J}$ (Version $1.50 \mathrm{i}, \mathrm{NIH}$, Washington, DC, USA). 
The amount of TBARS was estimated using the method described by Ramanathan and collaborators [26] with slight modifications. Culture medium was mixed with SDS (8\% w/v), thiobarbituric acid $\left(0.8 \%\right.$ TBA w/v), and acetic acid $(20 \% \mathrm{v} / \mathrm{v})$, and heated for $60 \mathrm{~min}$ at $90^{\circ} \mathrm{C}$. The material that had precipitated was removed by centrifugation, and the absorbance of the supernatant was evaluated at $532 \mathrm{~nm}$. The amount of TBARS was calculated using a calibration curve obtained with malondialdehyde (MDA) as standard. MDA was obtained from Merck (Darmstadt, Germany).

\section{Statistical Analysis}

Assuming values with normal distribution, results were evaluated by ANOVA test with a Sidak post-test of multiple comparisons. For non-normal data, we used non-parametric Kruskal-Wallis test with a Dunn post-test. Results below are expressed as the average of values from each independent experiment \pm SE and considered significantly different if $p<$ 0.05. Analyses were performed with the GraphPad Prism 9 software for Windows (19922020, GraphPad Software, La Jolla, CA, USA).

\section{Results}

The TNF- $\alpha / \mathrm{IL}-1 \beta$ induced activation of Cx43 HCs in MES-13 cells depends on RhoA/ROCK pathway.

We previously demonstrated that activation of $\mathrm{Cx} 43 \mathrm{HCs}$ evoked by Angll occurs at the same time as the high production of IL-1 $\beta$ and TNF- $\alpha$ in MES-13 cells [9]. However, whether both cytokines directly increase the activity of $\mathrm{Cx} 43 \mathrm{HCs}$ in these cells remains to be elucidated. For that purpose, the functional state of HCs was investigated by recording the rate of $\mathrm{Etd}^{+}$uptake. We found that under control conditions, MES-13 cells display a low Etd ${ }^{+}$ uptake. In contrast, the treatment for $72 \mathrm{~h}$ with TNF- $\alpha / \mathrm{IL}-1 \beta(10 \mathrm{ng} / \mathrm{ml})$ induced a 6 -fold increase in $\mathrm{Etd}^{+}$uptake compared to control values (from $3.2 \pm 1.00$ to $16.8 \pm 1.11 \mathrm{AU} / \mathrm{min}$ ) (Fig. 1A-B). No changes in $\mathrm{Etd}^{+}$uptake were observed in cells that were stimulated for shorter periods than $72 \mathrm{~h}$ or when TNF- $\alpha$ or IL-1 $\beta$ were added alone (Fig. 1C).

Since Cx43 HCs represent one of the most prevalent routes for dye influx in MES13 cells [9], the potential contribution of these channels in the TNF- $\alpha / I_{L}-1 \beta$-induced Etd ${ }^{+}$ uptake was examined. Accordingly, MES-13 cultures were pre-incubated for 15 min before and throughout Etd ${ }^{+}$uptake recordings with various pharmacological agents. We found that $200 \mu \mathrm{M} \mathrm{La}^{3+}$, a general blocker of Cx HCs [27], induced a drastic reduction in the Etd ${ }^{+}$uptake 
evoked by TNF- $\alpha / I L-1 \beta(5.6 \pm 0.72 \mathrm{AU} / \mathrm{min})$, whereas similar findings were observed upon the treatment with $250 \mu \mathrm{M}$ boldine $(3.9 \pm 0.41 \mathrm{AU} / \mathrm{min})$, another $\mathrm{Cx} \mathrm{HC}$ blocker [28]. Interestingly, $100 \mu \mathrm{M}$ Gap27, an inhibitory mimetic peptide with a sequence equivalent to a domain of the second extracellular L2 loop region of Cx43; completely blunted the TNF- $\alpha / \mathrm{IL}-$ $1 \beta$-induced Etd ${ }^{+}$uptake in MES-13 cells (2.9 $\left.\pm 0.56 \mathrm{AU} / \mathrm{min}\right)$ (Fig. 2). Overall, these results reveal that TNF- $\alpha / \mathrm{IL}-1 \beta$ enhances the function of $\mathrm{Cx} 43 \mathrm{HCs}$ in MCs.

To assess whether TNF- $\alpha / \mathrm{IL}-1 \beta$-induced opening of $\mathrm{Cx} 43 \mathrm{HCs}$ in MCs is mediated by a RhoA/ROCK-dependent pathway, we evaluated the effect of Fasudil and Y-27632two selective inhibitors of ROCK $[13,15,28,29]$. Similarly, pretreatment of MCs with Fasudil $(15 \mu \mathrm{M})$ or $\mathrm{Y}-27632(15 \mu \mathrm{M})$ strongly reduced the TNF- $\alpha / \mathrm{IL}-1 \beta$-induced activity of Cx43 HCs (Fasudil: $5.80 \pm 1.39 \mathrm{AU} / \mathrm{min}$; Y-27632: $5.70 \pm 1.44 \mathrm{AU} / \mathrm{min}$ ) (Fig. 2). The latter suggests that the increase in Cx43 HCs caused by TNF- $\alpha /$ IL-1 $\beta$ relies on ROCK activation in MCs.

TNF- $\alpha / I L-1 \beta$ reduces intercellular communication mediated by GJs in MES-13 cells.

Multiple lines of research have described that under pro-inflammatory conditions, the increased activity of $\mathrm{HCs}$ occurs in parallel with a decrease in gap junctional communication [30-32]. With this in mind, we decided to explore whether TNF- $\alpha /$ IL-1 $\beta$ could affect the functional state of GJs in MES-13 cells by measuring the intercellular diffusion of microinjected Etd ${ }^{+}$on single cells grown in clusters or monolayers [28,33].

$\mathrm{Etd}^{+}$intercellular coupling experiments revealed that under control conditions, almost $100 \%$ of MCs cells were coupled and most of them did it with $\sim 6$ neighboring cells (Fig. 3AC). It is worth noting that, $72 \mathrm{~h}$ of treatment with TNF- $\alpha / \mathrm{IL}-1 \beta$ caused a prominent reduction in the incidence of coupling (44 $\pm 6 \%$ ) (Fig. $3 \mathrm{C}$ ). In addition, the number of coupled cells (coupling index) decreased from $6.0 \pm 0.2$ cells to $2.0 \pm 0.2$ cells after treatment with TNF$\alpha / I L-1 \beta$ (Fig. 3B). Altogether these findings indicate that TNF- $\alpha / I L-1 \beta$ impacts the activity of HCs and GJ channels in an opposite manner. Surprisingly, pretreatment with $15 \mu \mathrm{M}$ Fasudil strongly prevented the inhibitory effect of TNF- $\alpha / \mathrm{IL}-1 \beta$ on gap junctional communication (incidence of coupling: $86.0 \pm 3.8 \%$; coupling index: $4.0 \pm 0.4$ cells) (Fig. 3A-C). No changes in cell-cell coupling were seen in cells treated with Fasudil alone (Fig. 3A). These findings reveal that the same metabolic pathway that increases the activity of HCs seems to be involved in the reduction of cell-cell communication mediated by GJs between MES-13 cells.

TNF- $\alpha / I L-1 \beta$ promotes phosphorylation of MYPT and increases the amount of Cx43 in mesangial cells. 
Given that TNF- $\alpha /$ IL-1 $\beta$ activates RhoA and Rho kinase (ROCK) [36] and alters Cx43 levels in different cell types [29,35,37], we decided to evaluate the activity of RhoA/ROCK and Cx43 protein levels. As such, we first measured the amount of phosphorylated MYPT-a downstream effector of the RhoA/ROCK pathway_and the relative amount of Cx43 in MES13 cells after treatment with TNF- $\alpha / I L-1 \beta$.

A clear increase in the relative amount of Cx43 was found in MES-13 cells after 72 $\mathrm{h}$ of treatment with TNF- $\alpha / \mathrm{IL}-1 \beta$ compared to control conditions. This response was partially inhibited by pretreatment with either Fasudil $(0.66 \pm 0.08 \mathrm{AU})$ or $\mathrm{Y}-27632(0.60 \pm 0.03 \mathrm{AU})$ (Fig. 4A, B). Similarly, $72 \mathrm{~h}$ of treatment with TNF- $\alpha / \mathrm{IL}-1 \beta$ also increased the phosphorylation of MYPT (1.00 $\pm 0.09 \mathrm{AU}$ ) compared to control conditions (Ctrl $0.25 \pm 0.02 \mathrm{AU}$ ), a response being partially suppressed by Fasudil or Y-27632 (0.57 $\pm 0.06 \mathrm{AU}$ or $0.50 \pm 0.09 \mathrm{AU})$ (Fig. 4C, D). Of note, neither Fasudil nor Y-27632 affected Cx43 levels or phosphorylation of MYPT in control cells (Fig. 4). Therefore, these data indicate that increases in phosphorylated MYPT and Cx43 levels evoked by TNF- $\alpha / \mathrm{IL}-1 \beta$ could be partly explained by the activation of RhoA/ROCK- pathway.

Inhibition of RhoA/ROCK prevents increases in lipid peroxidation responses induced by TNF- $\alpha$ and IL-1 $\beta$ in MES-13 Cells

TNF- $\alpha /$ IL-1 $\beta$ induces $\mathrm{Ca}^{2+}$ influx from the extracellular space and $\mathrm{Ca}^{2+}$ release from intracellular stores [34], leading to ROS generation and cell damage in several kidney diseases $[35,36]$. In addition, $\mathrm{HCs}$ regulate the cytosolic $\mathrm{Ca}^{2+}$ as they are permeable to this divalent cation or facilitate its intracellular increase by releasing ATP that activates purinergic receptors $[37,38]$. In this scenario, we evaluated whether TNF- $\alpha / I L-1 \beta$ could cause OS in MCs. We observed that the extracellular amount of TBARS increased $(1.1 \pm 0.04 \mu \mathrm{mol} / \mathrm{L})$ in MES-13 cells treated with TNF- $\alpha / \mathrm{IL}-1 \beta$ compared to control conditions $(0.11 \pm 0.03 \mu \mathrm{mol} / \mathrm{L})$ (Fig. 5). Moreover, when Fasudil or $\mathrm{Y}-27632$ was added to cells treated with TNF- $\alpha / \mathrm{IL}-1 \beta$, the extracellular amount of TBARS did increase significantly (Fasudil; $0.78 \pm 0.14 \mu \mathrm{mol} / \mathrm{L}$; $\mathrm{Y}-27632 ; 0.68 \pm 0.11 \mu \mathrm{mol} / \mathrm{L})$, as compared to what was found in control cell culture but did not increase significantly in the extracellular medium of cells treated only with TNF- $\alpha / \mathrm{IL}-1 \beta$ (Fig. 5). These data suggest that a RhoA/ROCK-dependent pathway does not prominently increase OS in MES-13 cells.

\section{Discussion}


The latest epidemiological study published in 2020 places CKD as the 12th cause of death worldwide and with a prevalence that increases each year [39], making this disease one of the leading public health problems today. It is essential therefore, to further understanding CKD's pathological mechanisms and to develop new therapeutic approaches [39]. In this work, we demonstrated that TNF- $\alpha /$ IL-1 $\beta$ increases Cx43 HC activity and simultaneously reduces gap junctional communication in MCs. Noticeably, the above response was strongly prevented by inhibiting the RhoA/ROCK pathway, indicating its crucial role in the TNF- $\alpha /$ IL$1 \beta$-mediated modulation of Cx-based channels in MES-13 cells. Of note, blockade of RhoA/ROCK pathway also reduces the TNF- $\alpha / I L-1 \beta$-induced production of OS. Based on this, we propose that RhoA/ROCK signaling contributes to the TNF- $\alpha / I L-1 \beta$-induced modulation of $\mathrm{HCs}$ and GJs with significant potentially negative consequences for the function and survival of MCs (Fig. 6)

MCs are pivotal for the normal functioning of the glomerulus [40]. These cells regulate intraglomerular capillary flow and the ultrafiltration surface due to their contractile properties and ability to respond to different substances. Among them are vasoactive molecules such as prostaglandin, adenosine, vasopressin, norepinephrine, Angll [40,41], and inflammatory mediators including IL-1 $\beta$, TNF- $\alpha$, and IFN- $\gamma$ [41]. In addition, MCs provide the structural support for the glomerular capillary network, and their crosstalk with other glomerular cell types, such as podocytes and endothelial cells, is fundamental for the proper function of the kidney [42]. Moreover, MCs play a role in the innate renal immune response as phagocytic cells by eliminating macromolecules, cells, and apoptotic bodies present in the mesangium [41]. Finally, MCs generate and control the turnover of the mesangial matrix in response to environmental cues $[43,44]$. Therefore, due to the multiple roles of MCs, they are considered a critical point in the origin and progression of various kidney diseases.

The pathophysiological mechanisms of kidney diseases are associated with factors that predispose to redox imbalance and the generation of inflammatory mediators, including ROS and TNF- $\alpha$ and IL-1 [45-47]. Inflammation is a well-known condition that reduces cellcell coupling but increases HC activity [48-50]. Consistent with this, the treatment with TNF$\alpha / \mathrm{IL}-1 \beta$ increased the Etd ${ }^{+}$uptake rate in MES-13 cells, a response being prevented by the blockade of Cx43 HCs with Gap27. Likewise, TNF- $\alpha$ /IL-1 $\beta$ also reduced GJ-mediated cellcell coupling and increased the amount of Cx43 protein in MES-13 cells. Previous studies have demonstrated that models of hypertensive-CKD and inflammation-CKD increase the expression of Cx43 in the early stage of CKD in the glomerulus [22], whereas increased HC activity occurs during heart attack and failure [51,52], neurodegenerative diseases [53], and 
liver fibrosis [54]. Several studies have shown that TNF- $\alpha / I L-1 \beta$ canonically activates the NFKB, a critical transcriptional factor that underpins the inflammatory response and has substantial consequences for redox balance as well [55]. Interestingly, the promoter of Cx43 contains a positive regulatory binding site for NFKB $[56,57]$. On the other hand, NFKB also induces the expression of iNOS and Cx43 via activation of PKA in MCs [58]. The latter would partially explain the increase in Cx43 protein observed in diseases with increased proinflammatory factors. Furthermore, nitric oxide (NO) can increase the opening probability of $\mathrm{Cx} 43 \mathrm{HCs}$ by S-nitrosylation of cysteine residues at the C-terminal, without altering their phosphorylation state [59].

RhoA/ROCK-dependent signaling pathways are critically involved in pathological conditions, including pulmonary hypertension [60], heart attack [61], stroke [62], Alzheimer's disease [63], glaucoma [64], diabetes and hypertensive nephropathy [65,66]. In addition, RhoA/ROCK is activated in MCs after stimulation with Angll [13]. Consistant with the above, we found an increase in levels of MYPT phosphorylated after treatment with TNF- $\alpha / I L-1 \beta$. These results are consistent with studies carried out in endothelial cells, where stimulation with TNF- $\alpha$ induces the activation of the RhoA/ROCK pathway by modulating the cytoskeleton and JNK-dependent secretion of IL-6 [67].

Intercellular communication in the kidney occurs directly via the cytoplasm of adjacent cells connected through GJs, and by paracrine signals released via large-pore channels, such as $\mathrm{HCs}$, pannexons and $\mathrm{P} 2 \mathrm{X}_{7} \mathrm{Rs}$ [68]. The release of ATP via HCs has multiple functions in the kidney, including regulating renal blood flow, glomerular filtration rate, and renal tubular transport [69]. However, the HC-mediated release of ATP and further activation of $\mathrm{P} 2 \mathrm{X}_{7} \mathrm{Rs}$ have been linked to inflammation and fibrosis $[68,70]$. Interestingly, our results show that inhibition of the RhoA/ROCK pathway prevent: i) the loss of GJ-mediated coupling, ii) the increase of $\mathrm{C} \times 43 \mathrm{HC}$ activity and iii) the augment in $\mathrm{C} x 43$ protein levels observed after TNF- $\alpha / \mathrm{IL}-1 \beta$ treatment. Therefore, the latter signaling is crucial for regulating the expression and function of Cx43-based channels in MCs during pro-inflammatory conditions. These results are consistent with previous evidence showing that the increased $\mathrm{HC}$ activity evoked by stimulation with Angll depends on activating a RhoA/ROCK signaling in MES-13 cells [9]. Relevantly, studies carried out in corneal epithelial cells in inflammatory conditions have shown that RhoA/ROCK signaling participates in the loss of cell-cell communication mediated by Cx43 GJ channels [71]. A similar effect has been observed in fibroblasts, where tissue stretching causes the opening of $\mathrm{Cx} 43 \mathrm{HCs}$ and the release of ATP through a mechanism mediated by RhoA/ROCK signaling [72]. On the contrary, thrombin- 
induced activation of RhoA GTPase controls extracellular purinergic signaling in endothelial cells by inhibiting Cx43 HCs [73]. Despite the apparent relationship between RhoA/ROCK signaling and $\mathrm{Cx} 43$, the use of Fasudil and Y-27632 did not reach the $\mathrm{Etd}^{+}$uptake values achieved by blocking of Cx43 HCs with Gap27. The latter could be due to the existence of additional mechanisms that would increase the activity of HCs in an independent-manner of RhoA/ROCK, such as the production of NO by iNOS [59].

The RhoA/ROCK-dependent signaling pathway has been extensively investigated in hypertensive pathology, where it plays a crucial role in regulating blood pressure and peripheral resistance [66]. In fact, treatment with Fasudil and Y-27632 has been suggested as an anti-hypertensive treatment given their hypotensive effect in DOCA-salt rat models [74] and spontaneously hypertensive rats [75]. Nevertheless, the NFKB pathway can also be activated by proteins of the Rho family: RhoA, Rac1 and Cdc42, which participate downstream of IL-1 $\beta$ and TNF- $\alpha[76,77]$. This work provides information to understand the possible molecular mechanisms underlying the dysfunction and damage of the kidney during pathological conditions and chronic diseases.

\section{Abbreviations}

Angll: Angiotensin II

AT1R: Angiotensin membrane G-protein-coupled receptors type I

AT2R: Angiotensin membrane G-protein-coupled receptors type II

CKD: Chronic kidney disease

Cx GJs: Connexin gap junctions

Cx HCs: Connexin hemichannels

Cx43: Connexin 43

ECM: Excess extracellular matrix

ESRD: End-stage renal disease

$\mathrm{Etd}^{+}$: Ethidium

IFN-Y: Interferon- $\mathrm{Y}$

IL-1 $\beta$ : Interleukin-1 $\beta$

IL-6: Interleukin-6

iNOS: inducible Nitric oxide synthase

MCs: Mesangial Cells

MDA: Malondialdehyde

MM: Mesangial matrix 
MYPT-1: Myosin phosphatase target subunit 1

NFkB: Nuclear factor kappa-light-chain-enhancer of activated $B$ cells

NO: Nitric oxide

OS: Oxidative stress

Panx1: Pannexin-1

RAS: Renin angiotensin system

Rho GTPase: Rho family of small GTPases

ROCK: Rho kinase

ROS: Reactive oxidative species

TBARS: Thiobarbituric reactive species

TNF- $\alpha$ : Tumor necrosis factor- $\alpha$

\section{Author Contributions}

G. I. G., V. V. and J. C. S. conceived and designed the experiments; G. I. G. performed the experiments; G. I. G, C. M. L. and M. L. analyzed the data; J. C. S., V. V and G. I. G. contributed reagents/materials/analysis tools; G. I. G., C. M. L., M. O., J. A. O, V. V., and J. C. S. wrote the paper.

\section{Funding}

Acknowledgments: This research was funded by CONICYT Ph.D. fellowship grant number 21120081 (to Gonzalo I. Gómez), FONDECYT grant number 1191329 (to Juan C. Sáez), FONDECYT grant number 1210375 (to Juan A. Orellana) and Iniciativa Científica Milenio (ICM)-ECONOMIA grant number P09-022-F from, Chile (to Juan C. Sáez) and FONDECYT INICIACIÓN grant number 11200584 (to Gonzalo I. Gómez).

\section{Conflicts of Interest}

The authors declare no conflict of interest.

\section{Figures}

Figure 1. TNF- $\alpha / \mathrm{IL}-1 \beta$ increases the ethidium uptake in mesangial cells. (A) Representative time-lapse recordings of $5 \mu \mathrm{M}$ ethidium $\left(\mathrm{Etd}^{+}\right)$uptake during $13 \mathrm{~min}$ in $\mathrm{MES}$ 13 cells under control conditions (black circles) or treated with TNF- $\alpha / \mathrm{lL}-1 \beta$ (10 $\mathrm{ng} / \mathrm{ml}$ each) for different periods (24-72 h). (B) Etd ${ }^{+}$uptake rate of MES-13 cells under control conditions (black bar) or after treatment with TNF- $\alpha / \mathrm{IL}-1 \beta$ for different periods (24-72 $\mathrm{h}$, white bar). (C) 
$\mathrm{Etd}^{+}$uptake rate of MES-13 cells under control conditions (black bar) or treated with TNF$\alpha / \mathrm{IL}-1 \beta 3$ (white bar), TNF- $\alpha$ (10 ng/ml, dashed bar) or IL-1 1 (10 ng/ml, gray bar) for $72 \mathrm{~h}$. Each bar represents the mean value \pm SE of five independent experiments with three replicates each one. Statistical significance * $p<0.05$ vs. Ctrl; $\uparrow p<0.05$ vs. (TNF- $\alpha /$ IL-1 $1 \beta$ ) $72 \mathrm{~h}$.

Figure 2. ROCK and Cx43 HC blockers reduce the TNF- $\alpha / \mathrm{IL}-1 \beta$-induced Etd ${ }^{+}$uptake in mesangial cells. Etd ${ }^{+}$uptake rate in MES-13 cells under control conditions (Ctrl, black bar), exposed to TNF- $\alpha / \mathrm{lL}-1 \beta$ (10 $\mathrm{ng} / \mathrm{ml}$ each, white bars) for $72 \mathrm{~h}$ alone or with ROCK inhibitors: Fasudil $(15 \mu \mathrm{M})$ and $\mathrm{Y}-27632(15 \mu \mathrm{M})$; HCs blockers: Lanthanum ion (La $\left.{ }^{3+}, 200 \mu \mathrm{M}\right)$ and Boldine (250 uM) or a selective Cx43 HC blocker: Mimetic peptide Gap27 (100 $\mu \mathrm{M})$. Each bar represents the mean value \pm SE of $=$ five independent experiments with three replicates each one. Statistical significance * $p<0.05$ vs. Ctrl; $\dagger p<0.05$ vs. TNF- $\alpha /$ IL-1 $\beta$. Boldine, Fasudil and Y-27632, were added during the last $24 \mathrm{~h}$ of treatment. Gap27 and $\mathrm{La}^{3+}$ were added acutely (added 15 min before each $\mathrm{Etd}^{+}$uptake recording).

Figure 3. Fasudil prevents the TNF- $\alpha / I L-1 \beta$-induced reduction in gap junctional communication in mesangial cells. (A) Gap junctional communication was examined using the microinjection of $\operatorname{Etd}^{+}(25 \mu \mathrm{M})$ in single cells (white arrows). Upon two min the diffusion to neighboring cells was imaged. The top panels represent phase-contrast images, whereas the bottom panels show $\mathrm{Etd}^{+}$fluorescence in cells under control conditions or upon different treatments. Scale bar $=20 \mu \mathrm{m}$. $(B)$ Coupling incidence and $(C)$ coupling index in confluent mesangial cells, under control conditions (black bars) or exposed to TNF- $\alpha / \mathrm{IL}-1 \beta$ $(10 \mathrm{ng} / \mathrm{ml})$ for $72 \mathrm{~h}$ (white bars). Fasudil $(15 \mu \mathrm{M})$ was added together with TNF- $\alpha / \mathrm{IL}-1 \beta$. Each bar represents the mean value \pm SE of 4 independent experiments. In each experiment, the dye was microinjected into at least 10 cells. Statistical significance * $p<0.05$ vs. Ctrl; $\uparrow p<$ 0.05 vs. TNF- $\alpha /$ IL-1 $\beta+$ Fasudil.

Figure 4. Blockade of ROCK prevents the reduction in connexin43 and phosphorylated MYPT in mesangial cells induced by TNF- $\alpha / I L-1 \beta$. (A) Graphs show the relative amount of $\mathrm{Cx} 43$ and $(C)$ phosphorylation of MYPT-1 determined by western blotting analysis in MES-13 cells under control conditions (black bars) or exposed to TNF- $\alpha / \mathrm{IL}-1 \beta$ (white bars) for $72 \mathrm{~h}$. Fasudil $(15 \mu \mathrm{M})$ or $\mathrm{Y}-27632(15 \mu \mathrm{M})$ were added $24 \mathrm{~h}$ before harvesting the cells. Each bar represents the mean value \pm SE of 4 independent experiments. Statistical 
significance * $p<0.05$ vs. Ctrl; \# $p<0.05$ vs. TNF- $\alpha /$ IL-1 $\beta$. Next to the graph, representative pictures of Cx43, phosphorylated MYPT (p-MYPT) unphosphorylated MYPT positive bands, and its loading control ( $\alpha$-tubulin) are shown.

Figure 5. Blockade of ROCK prevents the increase in TBARS induced by TNF- $\alpha /$ IL-1 $\beta$ in mesangial cells. Graphs showing the amount of TBARS obtained from culture media of MES-13 cells under control conditions (black bars) or exposed to TNF- $\alpha /$ IL-1 $\beta$ (white bars) for $72 \mathrm{~h}$. Fasudil $(15 \mu \mathrm{M})$ or $\mathrm{Y}-27632(15 \mu \mathrm{M})$ were added $24 \mathrm{~h}$ before harvesting the cells. Each bar represents the mean value \pm SE of 4 independent experiments. Statistical significance, ${ }^{*} p<0.05$ vs. Ctrl; \# $p<0.05$ vs. TNF- $\alpha /$ IL-1 $\beta$.

Figure 6. Schematics showing the possible signaling pathways involved in the TNF$\alpha / \mathrm{IL}-1 \beta$-mediated regulation of the functional state of $\mathrm{Cx} 43 \mathrm{HCs}$ and GJs in mesangial cells. High TNF- $\alpha /$ IL-1 $\beta$ concentrations could promote activation of a RhoA/ROCKdependent pathway through TNF- $\alpha / I L-1 \beta$ receptors (TNF-R and IL-1R) (continuous black arrows). Once the RhoA/ROCK-dependent pathway is activated, the formation of reactive oxygen species (ROS) that generate thiobarbituric acid reactive substances (TBARS) upon reaction with lipids of cell membranes can occur. The activated RhoA/ROCK-dependent pathway could increase the activity of $\mathrm{Cx} 43 \mathrm{HCs}$, since Fasudil or Y-27632 (ROCK blockers) inhibit this response. The resulting increase in intracellular $\mathrm{Ca}^{2+}$ will also activate $\mathrm{Cx} 43 \mathrm{HCs}$, allowing the increase in $\mathrm{Ca}^{2+}$ influx. The latter result in the rise of cytoplasmic $\mathrm{Ca}^{2+}$ concentration and further generation of ROS. The influx of $\mathrm{Ca}^{2+}$ establishes a positive feedback loop sensitive to different compounds such as boldine and the mimetic peptide Gap27, a selective Cx43 HCs blocker. This increase in the cellular activity caused by TNF$\alpha / I L-1 \beta$, where the RhoA/ROCK pathway could be involved, also reduces cell-cell communication through GJs. Discontinuous black arrows indicate cell responses identified in other systems, whereas straight black arrows denote responses identified in the present work. Elaborated in biorender.com

\section{References}

1. Yang, Z.-J.; Wang, H.-R.; Wang, Y.-I.; Zhai, Z.-H.; Wang, L.-W.; Li, L.; Zhang, C.;

Tang, L. Myricetin Attenuated Diabetes-Associated Kidney Injuries and Dysfunction 
via Regulating Nuclear Factor (Erythroid Derived 2)-Like 2 and Nuclear Factor-kB Signaling. Front. Pharmacol. 2019, 10, doi:10.3389/fphar.2019.00647.

2. Zhang, X.; Yang, J.; Yu, X.; Cheng, S.; Gan, H.; Xia, Y. Angiotensin II-Induced Early and Late Inflammatory Responses Through NOXs and MAPK Pathways. Inflammation 2017, 40, 154-165, doi:10.1007/s10753-016-0464-6.

3. Romagnani, P.; Remuzzi, G.; Glassock, R.; Levin, A.; Jager, K.J.; Tonelli, M.; Massy, Z.; Wanner, C.; Anders, H.-J. Chronic kidney disease. Nat. Rev. Dis. Prim. 2017, 3, 17088, doi:10.1038/nrdp.2017.88.

4. Zhao, J.-H. Mesangial Cells and Renal Fibrosis. In; 2019; pp. 165-194.

5. Kao, M.P.C.; Ang, D.S.C.; Pall, A.; Struthers, A.D. Oxidative stress in renal dysfunction: mechanisms, clinical sequelae and therapeutic options. J. Hum. Hypertens. 2010, 24, 1-8, doi:10.1038/jhh.2009.70.

6. Tucker, P.S.; Scanlan, A.T.; Dalbo, V.J. Chronic Kidney Disease Influences Multiple Systems: Describing the Relationship between Oxidative Stress, Inflammation, Kidney Damage, and Concomitant Disease. Oxid. Med. Cell. Longev. 2015, 2015, 1-8, doi:10.1155/2015/806358.

7. Alique, M.; Sánchez-López, E.; Rayego-Mateos, S.; Egido, J.; Ortiz, A.; RuizOrtega, M. Angiotensin II, via angiotensin receptor type 1/nuclear factor-kB activation, causes a synergistic effect on interleukin-1- $\beta$-induced inflammatory responses in cultured mesangial cells. J. Renin-Angiotensin-Aldosterone Syst. 2015, 16, 23-32, doi:10.1177/1470320314551564.

8. ZHANG, F.; SUN, D.; CHEN, J.; GUAN, N.; HUO, X.; XI, H. Simvastatin attenuates angiotensin II-induced inflammation and oxidative stress in human mesangial cells. Mol. Med. Rep. 2015, 11, 1246-1251, doi:10.3892/mmr.2014.2871.

9. Gómez, G.; Fernández, P.; Velarde, V.; Sáez, J. Angiotensin II-Induced Mesangial Cell Damage Is Preceded by Cell Membrane Permeabilization Due to Upregulation 
of Non-Selective Channels. Int. J. Mol. Sci. 2018, 19, 957, doi:10.3390/ijms19040957.

10. Gómez, G.; Velarde, V. Boldine Improves Kidney Damage in the Goldblatt 2K1C Model Avoiding the Increase in TGF- $\beta$. Int. J. Mol. Sci. 2018, 19, 1864, doi:10.3390/ijms19071864.

11. Gómez, G.I.; Velarde, V.; Sáez, J.C. Role of a RhoA/ROCK-Dependent Pathway on Renal Connexin43 Regulation in the Angiotensin II-Induced Renal Damage. Int. J. Mol. Sci. 2019, 20, 4408, doi:10.3390/ijms20184408.

12. da Silva Novaes, A.; Ribeiro, R.S.; Pereira, L.G.; Borges, F.T.; Boim, M.A. Intracrine action of angiotensin II in mesangial cells: subcellular distribution of angiotensin II receptor subtypes AT1 and AT2. Mol. Cell. Biochem. 2018, 448, 265-274, doi:10.1007/s11010-018-3331-y.

13. Gómez, G.I.; Velarde, V.; Sáez, J.C. Connexin-Based Channels and RhoA/ROCK Pathway in Angiotensin II-Induced Kidney Damage. In Renin-Angiotensin System; Gordan Tot, Ed.; 2019; pp. 1-16.

14. Sharma, N.; Anders, H.-J.; Gaikwad, A.B. Fiend and friend in the renin angiotensin system: An insight on acute kidney injury. Biomed. Pharmacother. 2019, 110, 764774, doi:10.1016/j.biopha.2018.12.018.

15. Rupérez, M.; Sánchez-López, E.; Blanco-Colio, L.M.; Esteban, V.; Rodríguez-Vita, J.; Plaza, J.J.; Egido, J.; Ruiz-Ortega, M. The Rho-kinase pathway regulates angiotensin II-induced renal damage. Kidney Int. Suppl. 2005, 68, S39-45, doi:10.1111/j.1523-1755.2005.09908.x.

16. Guilluy, C.; Rolli-Derkinderen, M.; Loufrani, L.; Bourgé, A.; Henrion, D.; Sabourin, L.; Loirand, G.; Pacaud, P. Ste20-related kinase SLK phosphorylates Ser188 of RhoA to induce vasodilation in response to angiotensin II Type 2 receptor activation. Circ. Res. 2008, 102, 1265-74, doi:10.1161/CIRCRESAHA.107.164764. 
17. Rao, J.; Ye, Z.; Tang, H.; Wang, C.; Peng, H.; Lai, W.; Li, Y.; Huang, W.; Lou, T. The RhoA/ROCK Pathway Ameliorates Adhesion and Inflammatory Infiltration Induced by AGEs in Glomerular Endothelial Cells. Sci. Rep. 2017, 7, 39727, doi:10.1038/srep39727.

18. Ye, Q.; Zhao, S.; Zhang, Y.; Su, Y.-M.; Chen, M.; Zhao, J.; Jia, G.-Z.; Han, B.-M.; Jiang, J.-T. Activation of the RhoA/ROCK pathway contributes to renal fibrosis in offspring rats induced by maternal exposure to di-n-butyl phthalate. Toxicology 2020, 443, 152573, doi:10.1016/j.tox.2020.152573.

19. BABA, I.; EGI, Y.; UTSUMI, H.; KAKIMOTO, T.; SUZUKI, K. Inhibitory effects of fasudil on renal interstitial fibrosis induced by unilateral ureteral obstruction. Mol. Med. Rep. 2015, 12, 8010-8020, doi:10.3892/mmr.2015.4467.

20. Sáez, J.C.; Contreras-Duarte, S.; Gómez, G.I.; Labra, V.C.; Santibañez, C.A.; Gajardo-Gómez, R.; Avendaño, B.C.; Díaz, E.F.; Montero, T.D.; Velarde, V.; et al. Connexin 43 hemichannel activity promoted by pro-inflammatory cytokines and high glucose alters endothelial cell function. Front. Immunol. 2018, 9, doi:10.3389/fimmu.2018.01899.

21. Prakoura, N.; Kavvadas, P.; Chadjichristos, C.E. Connexin 43: a New Therapeutic Target Against Chronic Kidney Disease. Cell. Physiol. Biochem. 2018, 49, 985-985, doi:10.1159/000493230.

22. Toubas, J.; Beck, S.; Pageaud, A.-L.; Huby, A.-C.; Mael-Ainin, M.; Dussaule, J.-C.; Chatziantoniou, C.; Chadjichristos, C.E. Alteration of connexin expression is an early signal for chronic kidney disease. Am. J. Physiol. Renal Physiol. 2011, 301, F24-32, doi:10.1152/ajprenal.00255.2010.

23. Orellana, J.A.; Sáez, P.J.; Cortés-Campos, C.; Elizondo, R.J.; Shoji, K.F.; Contreras-Duarte, S.; Figueroa, V.; Velarde, V.; Jiang, J.X.; Nualart, F.; et al. Glucose increases intracellular free $\mathrm{Ca}(2+)$ in tanycytes via ATP released through 
connexin 43 hemichannels. Glia 2012, 60, 53-68, doi:10.1002/glia.21246.

24. Contreras, J.E.; Sanchez, H.A.; Eugenin, E.A.; Speidel, D.; Theis, M.; Willecke, K.; Bukauskas, F.F.; Bennett, M.V.L.; Saez, J.C. Metabolic inhibition induces opening of unapposed connexin 43 gap junction hemichannels and reduces gap junctional communication in cortical astrocytes in culture. Proc. Natl. Acad. Sci. 2002, 99, 495-500, doi:10.1073/pnas.012589799.

25. LOWRY, O.H.; ROSEBROUGH, N.J.; FARR, A.L.; RANDALL, R.J. Protein measurement with the Folin phenol reagent. J. Biol. Chem. 1951, 193, 265-75.

26. Ramanathan, L.; Das, N.P.; Li, Q.T. Studies on lipid oxidation in fish phospholipid liposomes. Biol. Trace Elem. Res. 1994, 40, 59-70.

27. Nakazawa, K.; Liu, M.; Inoue, K.; Ohno, Y. Potent inhibition by trivalent cations of ATP-gated channels. Eur. J. Pharmacol. 1997, 325, 237-243, doi:10.1016/S00142999(97)00120-9.

28. Gómez, G.I.; Fernández, P.; Velarde, V.; Sáez, J.C. Angiotensin II-induced mesangial cell damaged is preceded by cell membrane permeabilization due to upregulation of non-selective channels. Int. J. Mol. Sci. 2018, 19, doi:10.3390/ijms19040957.

29. Kushiyama, T.; Oda, T.; Yamamoto, K.; Higashi, K.; Watanabe, A.; Takechi, H.; Uchida, T.; Oshima, N.; Sakurai, Y.; Miura, S.; et al. Protective effects of Rho kinase inhibitor fasudil on rats with chronic kidney disease. Am. J. Physiol. Renal Physiol. 2013, 304, F1325-34, doi:10.1152/ajprenal.00447.2012.

30. Retamal, M.A.; Froger, N.; Palacios-Prado, N.; Ezan, P.; Sáez, P.J.; Sáez, J.C.; Giaume, C. Cx43 hemichannels and gap junction channels in astrocytes are regulated oppositely by proinflammatory cytokines released from activated microglia. J. Neurosci. 2007, 27, 13781-92, doi:10.1523/JNEUROSCI.204207.2007 . 
31. Orellana, J.A.; Hernández, D.E.; Ezan, P.; Velarde, V.; Bennett, M.V.L.; Giaume, C.; Sáez, J.C. Hypoxia in high glucose followed by reoxygenation in normal glucose reduces the viability of cortical astrocytes through increased permeability of connexin 43 hemichannels. Glia 2010, 58, 329-43, doi:10.1002/glia.20926.

32. Hernández-Salinas, R.; Vielma, A.Z.; Arismendi, M.N.; Boric, M.P.; Sáez, J.C.; Velarde, V. Boldine Prevents Renal Alterations in Diabetic Rats. J. Diabetes Res. 2013, 2013, 1-12, doi:10.1155/2013/593672.

33. Saez, J.C.; Berthoud, V.M.; Branes, M.C.; Martinez, A.D.; Beyer, E.C. Plasma membrane channels formed by connexins: their regulation and functions. Physiol. Rev. 2003, 83, 1359-400, doi:10.1152/physrev.00007.2003.

34. Zhong, G.; Long, H.; Fei, C.; Yu, Y. Oxoglaucine mediates Ca 2+ influx and activates autophagy to alleviate osteoarthritis through the TRPV5/Calmodulin/CAMK-II pathway. Br. J. Pharmacol. 2021, bph.15466, doi:10.1111/bph.15466.

35. Peng, F.; Wu, D.; Gao, B.; Ingram, A.J.; Zhang, B.; Chorneyko, K.; McKenzie, R.; Krepinsky, J.C. RhoA/Rho-kinase contribute to the pathogenesis of diabetic renal disease. Diabetes 2008, 57, 1683-92, doi:10.2337/db07-1149.

36. Zhao, W.; Chen, S.S.; Chen, Y.; Ahokas, R.A.; Sun, Y. Kidney fibrosis in hypertensive rats: role of oxidative stress. Am. J. Nephrol. 2008, 28, 548-54, doi:10.1159/000115289.

37. Schalper, K.A.; Sánchez, H.A.; Lee, S.C.; Altenberg, G.A.; Nathanson, M.H.; Sáez, J.C. Connexin 43 hemichannels mediate the Ca $2+$ influx induced by extracellular alkalinization. Am. J. Physiol. Physiol. 2010, 299, C1504-C1515, doi:10.1152/ajpcell.00015.2010.

38. Fiori, M.C.; Figueroa, V.; Zoghbi, M.E.; Saéz, J.C.; Reuss, L.; Altenberg, G.A. Permeation of Calcium through Purified Connexin 26 Hemichannels. J. Biol. Chem. 
2012, 287, 40826-40834, doi:10.1074/jbc.M112.383281.

39. Bikbov, B.; Purcell, C.A.; Levey, A.S.; Smith, M.; Abdoli, A.; Abebe, M.; Adebayo, O.M.; Afarideh, M.; Agarwal, S.K.; Agudelo-Botero, M.; et al. Global, regional, and national burden of chronic kidney disease, 1990-2017: a systematic analysis for the Global Burden of Disease Study 2017. Lancet 2020, 395, 709-733, doi:10.1016/S0140-6736(20)30045-3.

40. STOCKAND, J.D.; SANSOM, S.C. Glomerular Mesangial Cells: Electrophysiology and Regulation of Contraction. Physiol. Rev. 1998, 78, 723-744, doi:10.1152/physrev.1998.78.3.723.

41. Schlöndorff, D.; Banas, B. The Mesangial Cell Revisited: No Cell Is an Island. J. Am. Soc. Nephrol. 2009, 20, 1179-1187, doi:10.1681/ASN.2008050549.

42. Vaughan, M.R.; Quaggin, S.E. How Do Mesangial and Endothelial Cells Form the Glomerular Tuft? J. Am. Soc. Nephrol. 2008, 19, 24-33, doi:10.1681/ASN.2007040471.

43. Couchman, J.R.; Beavan, L.A.; McCarthy, K.J. Glomerular matrix: Synthesis, turnover and role in mesangial expansion. Kidney Int. 1994, 45, 328-335, doi:10.1038/ki.1994.42.

44. Mason, R.M.; Wahab, N.A. Extracellular Matrix Metabolism in Diabetic Nephropathy. J. Am. Soc. Nephrol. 2003, 14, 1358-1373, doi:10.1097/01.ASN.0000065640.77499.D7.

45. Kao, M.P.C.; Ang, D.S.C.; Pall, A.; Struthers, A.D. Oxidative stress in renal dysfunction: mechanisms, clinical sequelae and therapeutic options. J. Hum. Hypertens. 2010, 24, 1-8, doi:10.1038/jhh.2009.70.

46. Bao, L.; Locovei, S.; Dahl, G. Pannexin membrane channels are mechanosensitive conduits for ATP. FEBS Lett. 2004, 572, 65-68, doi:10.1016/j.febslet.2004.07.009.

47. Singh, P.; Bahrami, L.; Castillo, A.; Majid, D.S.A. TNF- $\alpha$ type 2 receptor mediates 
renal inflammatory response to chronic angiotensin II administration with high salt intake in mice. Am. J. Physiol. Renal Physiol. 2013, 304, F991-9, doi:10.1152/ajprenal.00525.2012.

48. González, H.E.; Eugenín, E.A.; Garcés, G.; Solís, N.; Pizarro, M.; Accatino, L.; Sáez, J.C. Regulation of hepatic connexins in cholestasis: possible involvement of Kupffer cells and inflammatory mediators. Am. J. Physiol. Liver Physiol. 2002, 282, G991-G1001, doi:10.1152/ajpgi.00298.2001.

49. Haefliger, J.-A.; Krattinger, N.; Martin, D.; Pedrazzini, T.; Capponi, A.; Döring, B.; Plum, A.; Charollais, A.; Willecke, K.; Meda, P. Connexin43-dependent mechanism modulates renin secretion and hypertension. J. Clin. Invest. 2006, 116, 405-13, doi:10.1172/JCI23327.

50. Même, W.; Calvo, C.; Froger, N.; Ezan, P.; Amigou, E.; Koulakoff, A.; Giaume, C. Proinflammatory cytokines released from microglia inhibit gap junctions in astrocytes: potentiation by $\beta$-amyloid. FASEB J. 2006, 20, 494-496, doi:10.1096/fj.05-4297fje.

51. Johansen, D.; Cruciani, V.; Sundset, R.; Ytrehus, K.; Mikalsen, S.-O. Ischemia Induces Closure of Gap Junctional Channels and Opening of Hemichannels in Heart-derived Cells and Tissue. Cell. Physiol. Biochem. 2011, 28, 103-114, doi:10.1159/000331719.

52. Lucero, C.M.; Andrade, D.C.; Toledo, C.; Díaz, H.S.; Pereyra, K. V.; Diaz-Jara, E.; Schwarz, K.G.; Marcus, N.J.; Retamal, M.A.; Quintanilla, R.A.; et al. Cardiac remodeling and arrhythmogenesis are ameliorated by administration of Cx43 mimetic peptide Gap27 in heart failure rats. Sci. Rep. 2020, 10, 6878, doi:10.1038/s41598-020-63336-6.

53. Orellana, J.A.; von Bernhardi, R.; Giaume, C.; Sáez, J.C. Glial hemichannels and their involvement in aging and neurodegenerative diseases. Rev. Neurosci. 2012, 
23, 163-77, doi:10.1515/revneuro-2011-0065.

54. Crespo Yanguas, S.; da Silva, T.; Pereira, I.; Willebrords, J.; Maes, M.; Sayuri Nogueira, M.; Alves de Castro, I.; Leclercq, I.; Romualdo, G.; Barbisan, L.; et al. TAT-Gap19 and Carbenoxolone Alleviate Liver Fibrosis in Mice. Int. J. Mol. Sci. 2018, 19, 817, doi:10.3390/ijms19030817.

55. Perkins, N.D. The diverse and complex roles of NF-kB subunits in cancer. Nat. Rev. Cancer 2012, 12, 121-132, doi:10.1038/nrc3204.

56. Alonso, F.; Krattinger, N.; Mazzolai, L.; Simon, A.; Waeber, G.; Meda, P.; Haefliger, J.-A. An angiotensin II- and NF-KB-dependent mechanism increases connexin 43 in murine arteries targeted by renin-dependent hypertension. Cardiovasc. Res. 2010, 87, 166-176, doi:10.1093/cvr/cvq031.

57. Zhao, Y.; Rivieccio, M.A.; Lutz, S.; Scemes, E.; Brosnan, C.F. The TLR3 ligand polyl:C downregulates connexin 43 expression and function in astrocytes by a mechanism involving the NF-KB and PI3 kinase pathways. Glia 2006, 54, 775-785, doi:10.1002/glia.20418.

58. Yao, J.; Hiramatsu, N.; Zhu, Y.; Morioka, T.; Takeda, M.; Oite, T.; Kitamura, M. Nitric Oxide-Mediated Regulation of Connexin43 Expression and Gap Junctional Intercellular Communication in Mesangial Cells. J. Am. Soc. Nephrol. 2005, 16, 5867, doi:10.1681/ASN.2004060453.

59. Retamal, M.A.; Cortes, C.J.; Reuss, L.; Bennett, M.V.L.; Saez, J.C. S-nitrosylation and permeation through connexin 43 hemichannels in astrocytes: Induction by oxidant stress and reversal by reducing agents. Proc. Natl. Acad. Sci. 2006, 103, 4475-4480, doi:10.1073/pnas.0511118103.

60. Jin, L.; Ying, Z.; Hilgers, R.H.P.; Yin, J.; Zhao, X.; Imig, J.D.; Webb, R.C. Increased RhoA/Rho-Kinase Signaling Mediates Spontaneous Tone in Aorta from Angiotensin II-Induced Hypertensive Rats. J. Pharmacol. Exp. Ther. 2006, 318, 288-295, 
doi:10.1124/jpet.105.100735.

61. Hamid, S.A.; Bower, H.S.; Baxter, G.F. Rho kinase activation plays a major role as a mediator of irreversible injury in reperfused myocardium. Am. J. Physiol. Circ. Physiol. 2007, 292, H2598-H2606, doi:10.1152/ajpheart.01393.2006.

62. Rikitake, Y.; Kim, H.-H.; Huang, Z.; Seto, M.; Yano, K.; Asano, T.; Moskowitz, M.A.; Liao, J.K. Inhibition of Rho Kinase (ROCK) Leads to Increased Cerebral Blood Flow and Stroke Protection. Stroke 2005, 36, 2251-2257, doi:10.1161/01.STR.0000181077.84981.11.

63. Mueller, B.K.; Mack, H.; Teusch, N. Rho kinase, a promising drug target for neurological disorders. Nat. Rev. Drug Discov. 2005, 4, 387-398, doi:10.1038/nrd1719.

64. Honjo, M.; Tanihara, H.; Inatani, M.; Kido, N.; Sawamura, T.; Yue, B.Y.; Narumiya, S.; Honda, Y. Effects of rho-associated protein kinase inhibitor Y-27632 on intraocular pressure and outflow facility. Invest. Ophthalmol. Vis. Sci. 2001, 42, 137-44.

65. Xie, X.; Peng, J.; Chang, X.; Huang, K.; Huang, J.; Wang, S.; Shen, X.; Liu, P.; Huang, $H$. Activation of RhoA/ROCK regulates NF-KB signaling pathway in experimental diabetic nephropathy. Mol. Cell. Endocrinol. 2013, 369, 86-97, doi:10.1016/j.mce.2013.01.007.

66. Lai, A.; Frishman, W.H. Rho-kinase inhibition in the therapy of cardiovascular disease. Cardiol. Rev. 2005, 13, 285-92.

67. Mong, P.Y.; Petrulio, C.; Kaufman, H.L.; Wang, Q. Activation of Rho Kinase by TNFa Is Required for JNK Activation in Human Pulmonary Microvascular Endothelial Cells. J. Immunol. 2008, 180, 550-558, doi:10.4049/jimmunol.180.1.550.

68. Price, G.W.; Potter, J.A.; Williams, B.M.; Cliff, C.L.; Squires, P.E.; Hills, C.E. Connexin-mediated cell communication in the kidney: A potential therapeutic target 
for future intervention of diabetic kidney disease? Exp. Physiol. 2020, 105, 219-229, doi:10.1113/EP087770.

69. Burnstock, G.; Arnett, T.R.; Orriss, I.R. Purinergic signalling in the musculoskeletal system. Purinergic Signal. 2013, 9, 541-572, doi:10.1007/s11302-013-9381-4.

70. Becker, L.V.; Passos, D.F.; Leal, D.B.R.; Morsch, V.M.; Schetinger, M.R.C. ATP signaling and NTPDase in Systemic Lupus Erythematosus (SLE). Immunobiology 2019, 224, 419-426, doi:10.1016/j.imbio.2019.02.005.

71. Anderson, S.C.; Stone, C.; Tkach, L.; SundarRaj, N. Rho and Rho-kinase (ROCK) signaling in adherens and gap junction assembly in corneal epithelium. Invest. Ophthalmol. Vis. Sci. 2002, 43, 978-86.

72. Langevin, H.M.; Fujita, T.; Bouffard, N.A.; Takano, T.; Koptiuch, C.; Badger, G.J.; Nedergaard, M. Fibroblast cytoskeletal remodeling induced by tissue stretch involves ATP signaling. J. Cell. Physiol. 2013, 228, 1922-6, doi:10.1002/jcp.24356.

73. Ponsaerts, R.; D’hondt, C.; Hertens, F.; Parys, J.B.; Leybaert, L.; Vereecke, J.; Himpens, B.; Bultynck, G. RhoA GTPase Switch Controls Cx43-Hemichannel Activity through the Contractile System. PLoS One 2012, 7, e42074, doi:10.1371/journal.pone.0042074.

74. Uehata, M.; Ishizaki, T.; Satoh, H.; Ono, T.; Kawahara, T.; Morishita, T.; Tamakawa, H.; Yamagami, K.; Inui, J.; Maekawa, M.; et al. Calcium sensitization of smooth muscle mediated by a Rho-associated protein kinase in hypertension. Nature 1997, 389, 990-994, doi:10.1038/40187.

75. Mukai, Y.; Shimokawa, H.; Matoba, T.; Kandabashi, T.; Satoh, S.; Hiroki, J.; Kaibuchi, K.; Takeshita, A. Involvement of Rho-kinase in hypertensive vascular disease: a novel therapeutic target in hypertension. FASEB J. 2001, 15, 1062-4.

76. Perona, R.; Montaner, S.; Saniger, L.; Sanchez-Perez, I.; Bravo, R.; Lacal, J.C. Activation of the nuclear factor-kappaB by Rho, CDC42, and Rac-1 proteins. Genes 
Dev. 1997, 11, 463-475, doi:10.1101/gad.11.4.463.

77. Wong, E.T.; Tergaonkar, V. Roles of NF-KB in health and disease: mechanisms and therapeutic potential. Clin. Sci. 2009, 116, 451-465, doi:10.1042/CS20080502. 